\title{
An improved spectral homotopy analysis method for solving boundary layer problems
}

Sandile Sydney Motsa ${ }^{1}$, Gerald T Marewo ${ }^{1}$, Precious Sibanda ${ }^{2}$ and Stanford Shateyi ${ }^{3^{*}}$

* Correspondence: stanford.

shateyi@univen.ac.za

${ }^{3}$ Department of Mathematics,

University of Venda, Private Bag

X5050, Thohoyandou 0950, South

Africa

Full list of author information is available at the end of the article

\begin{abstract}
This article presents an improved spectral-homotopy analysis method (ISHAM) for solving nonlinear differential equations. The implementation of this new technique is shown by solving the Falkner-Skan and magnetohydrodynamic boundary layer problems. The results obtained are compared to numerical solutions in the literature and MATLAB's bvp $4 \mathrm{C}$ solver. The results show that the ISHAM converges faster and gives accurate results.
\end{abstract}

Keywords: Falkner-Skan flow, MHD flow, improved spectral-homotopy analysis method

\section{Introduction}

Boundary layer flow problems have wide applications in fluid mechanics. In this article, we propose an improved spectral-homotopy analysis method (ISHAM) for solving general boundary layer problems. Three boundary layer problems are considered and solved in this study using the novel technique. The first problem considered is the classical two-point nonlinear boundary value Blasius problem which models viscous fluid flow over a semi-infinite flat plate. Although solutions for this problem had been obtained as far back as 1908 by Blasius [1], the problem is still of great interest to many researchers as can be seen from the several recent studies [2-5].

The second problem considered in this article is the third-order nonlinear FalknerSkan equation. The Falkner-Skan boundary layer equation has been studied by several researchers from as early as 1931 [6]. More recent studies of the solutions of the The Falkner-Skan equation include those of Harries et al. [7], Pade [8] and Pantokratoras [9]. The third problem considered is magnetohy-drodynamic (MHD) boundary layer flow. Such boundary layer problems arise in the study of the flow of electrically conducting fluids such as liquid metal. Owing to its many applications such as power generators, flow meters, and the cooling of reactors, MHD flow has been studied by many researchers, for example $[10,11]$.

Owing to the nonlinearity of equations that describe most engineering and science phenomena, many authors traditionally resort to numerical methods such as finite difference methods [12], Runge-Kutta methods [13], finite element methods [14] and spectral methods [4] to solve the governing equations. However, in recent years, several analytical or semi-analytical methods have been proposed and used to find solutions to most nonlinear equations. These methods include the Adomian

(c) 2011 Motsa et al; licensee Springer. This is an Open Access article distributed under the terms of the Creative Commons Attribution License (http://creativecommons.org/licenses/by/2.0), which permits unrestricted use, distribution, and reproduction in any medium, provided the original work is properly cited. 
decomposition method [15-17], differential transform method [18], variational iteration method [19], homotopy analysis method (HAM) [20-23], and the spectral-homotopy analysis (SHAM) (see Motsa et al. $[24,25]$ ) which sought to remove some of the perceived limitations of the HAM. More recently, successive linearization method [26-28], has been used successfully to solve nonlinear equations that govern the flow of fluids in bounded domains.

In this article, boundary layer equations are solved using the ISHAM. The ISHAM is a modified version of the SHAM $[24,25]$. One strength of the SHAM is that it removes restrictions of the HAM such as the requirement for the solution to conform to the so-called rule of solution expression and the rule of coefficient ergodicity. Also, the SHAM inherits the strengths of the HAM, for example, it does not depend on the existence of a small parameter in the equation to be solved, it avoids discretization, and the solution obtained is in terms of an auxiliary parameter $\hbar$ which can conveniently be chosen to determine the convergence rate of the solution.

\section{Mathematical formulation}

We consider the general nonlinear third-order boundary value problem

$$
f^{\prime \prime \prime}+c_{1} f f^{\prime \prime}+c_{2}\left(f^{\prime}\right)^{2}+c_{3} f^{\prime}+c_{4}=0,
$$

subject to the boundary conditions

$$
f(0)=b_{1}, \quad f^{\prime}(0)=b_{2}, \quad f^{\prime}(\infty)=b_{3},
$$

where $c_{i}, b_{j}(i=1, \ldots, 4 j=1,2,3)$ are constants.

Equation 2.1 can be solved easily using methods such as the HAM and the SHAM. In each of these methods, an initial approximation $f_{0}(\eta)$ is sought, which satisfies the boundary conditions. The speed of convergence of the method depends on whether $f_{0}$ $(\eta)$ is a good approximation of $f(\eta)$ or not. The approach proposed here seeks to find an optimal initial approximation $f_{0}$ that would lead to faster convergence of the method to the true solution. We thus first seek to improve the initial approximation that is used later in the SHAM to solve the governing nonlinear equation.

We assume that the solution $f(\eta)$ may be expanded as an infinite sum:

$$
f(\eta)=f_{i}(\eta)+\sum_{n=0}^{i-1} f_{n}(\eta), \quad i=1,2,3, \ldots
$$

where $f_{i}^{\prime}$ 's are unknown functions whose solutions are obtained using the SHAM at the $i$ th iteration and $f_{n},(n \geq 1)$ are known from previous iterations. The algorithm starts with the initial approximation $f_{0}(\eta)$ which is chosen to satisfy the boundary conditions (2.2). An appropriate initial guess is

$$
f_{0}(\eta)=b_{3} \eta-\left(b_{2}-b_{3}\right) e^{-\eta}+b_{1}+b_{2}-b_{3} .
$$

Substituting (2.3) in the governing equation (2.1-2.2) gives

$$
f_{i}^{\prime \prime \prime}+a_{1, i-1} f_{i}^{\prime \prime}+a_{2, i-1} f_{i}^{\prime}+a_{3, i-1} f_{i}+c_{1} f_{i}^{\prime \prime} f_{i}+c_{2}\left(f_{i}^{\prime}\right)^{2}=r_{i-1},
$$

subject to the boundary conditions

$$
f_{i}(0)=0, \quad f_{i}^{\prime}(0)=0, \quad f_{i}^{\prime}(\infty)=0,
$$


where the coefficient parameters $a_{k, i-1},(k=1, \ldots, 3)$ and $r_{i-1}$ are defined as

$$
\begin{aligned}
& a_{1, i-1}=c_{1} \sum_{n=0}^{i-1} f_{n}, \quad a_{2, i-1}=2 c_{2} \sum_{n=0}^{i-1} f_{n}^{\prime}+c_{3}, \quad a_{3, i-1}=c_{1} \sum_{n=0}^{i-1} f_{n}^{\prime \prime}, \\
& r_{i-1}=-\left[\sum_{n=0}^{i-1} f_{n}^{\prime \prime \prime}+c_{1} \sum_{n=0}^{i-1} f_{n}^{\prime \prime} \sum_{n=0}^{i-1} f_{n}+c_{2}\left(\sum_{n=0}^{i-1} f_{n}^{\prime}\right)^{2}+c_{3} \sum_{n=0}^{i-1} f_{n}^{\prime}+c_{4}\right] .
\end{aligned}
$$

Starting from the initial approximation (2.4), the subsequent solutions $f_{i}(i \geq 1)$ are obtained by recursively solving Equation 2.5 using the SHAM, [24,25]. To find the solutions of Equation 2.5, we begin by defining the following linear operator:

$$
\mathcal{L}\left[F_{i}(\eta ; q)\right]=\frac{\partial^{3} F_{i}}{\partial \eta^{3}}+a_{1, i-1} \frac{\partial^{2} F_{i}}{\partial \eta^{2}}+a_{2, i-1} \frac{\partial F_{i}}{\partial \eta}+a_{3, i-1} F_{i}
$$

where $q \in 0[1]$ is the embedding parameter, and $F_{i}(\eta ; q)$ is an unknown function.

The zeroth-order deformation equation is given by

$$
(1-q) \mathcal{L}\left[F_{i}(\eta ; q)-f_{i, 0}(\eta)\right]=q \hbar\left\{\mathcal{N}\left[F_{i}(\eta ; q)\right]-r_{i-1}\right\} .
$$

where $\hbar$ is the non-zero convergence controlling auxiliary parameter and $\mathcal{N}$ is a nonlinear operator given by

$$
\mathcal{N}\left[F_{i}(\eta ; q)\right]=\frac{\partial^{3} F_{i}}{\partial \eta^{3}}+a_{1, i-1} \frac{\partial^{2} F_{i}}{\partial \eta^{2}}+a_{2, i-1} \frac{\partial F_{i}}{\partial \eta}+a_{3, i-1} F_{i}+c_{1} F_{i} \frac{\partial^{2} F_{i}}{\partial \eta^{2}}+c_{2}\left[\frac{\partial F_{i}}{\partial \eta}\right]^{2}
$$

Differentiating (2.10) $m$ times with respect to $q$ and then setting $q=0$, and finally dividing the resulting equations by $m$ ! yield the $m$ th-order deformation equations:

$$
\begin{aligned}
& \mathcal{L}\left[f_{i, m}(\eta)-\chi_{m} f_{i, m-1}\right]=\hbar\left(f_{i, m-1}^{\prime \prime \prime}+a_{1, i-1} f_{i, m-1}^{\prime \prime}+a_{2, i-1} f_{i, m-1}^{\prime}+a_{3, i-1} f_{i, m-1}\right. \\
& \left.+c_{1} \sum_{j=0}^{m-1} f_{i, j} f_{i, m-1-j}^{\prime \prime}+c_{2} \sum_{j=0}^{m-1} f_{i, j}^{\prime} f_{i, m-1-j}^{\prime}-\left(1-\chi_{m}\right) r_{i-1}\right),
\end{aligned}
$$

subject to the boundary conditions

$$
f_{i, m}(0)=f_{i, m}^{\prime}(0)=f_{i, m}^{\prime}(\infty)=0,
$$

where

$$
\chi_{m}=\left\{\begin{array}{l}
0, m \leq 1 \\
1, m>1
\end{array} .\right.
$$

The initial approximation $f_{i, 0}$ that is used in the higher-order equations (2.12) is obtained on solving the linear part of Equation 2.5 which is given by

$$
f_{i, 0}^{\prime \prime \prime}+a_{1, i-1} f_{i, 0}^{\prime \prime}+a_{2, i-1} f_{i, 0}^{\prime}+a_{3, i-1} f_{i, 0}=r_{i-1}
$$

subject to the boundary conditions:

$$
f_{i, 0}(0)=f_{i, 0}^{\prime}(0)=f_{i, 0}^{\prime}(\infty)=0
$$


Since the coefficient parameters and the right-hand side of Equation 2.15 for $i=1,2$, $3, \ldots$ are known (from previous iterations), the equation can easily be solved using numerical methods such as finite differences, finite elements, Runge-Kutta-based shooting methods or collocation methods. In this article, Equation 2.15 are solved using the Chebyshev spectral collocation method. The method (see, for example, [29-31]), is based on the Chebyshev polynomials defined on the interval $[-1,1]$ by

$$
T_{k}(\xi)=\cos \left[k \cos ^{-1}(\xi)\right] .
$$

To implement the method, the physical region $[0, \infty)$ is transformed into the region $[-1,1]$ using the domain truncation technique whereby the problem is solved in the interval $[0, L]$ instead of $[0, \infty)$. This leads to the mapping

$$
\frac{\eta}{L}=\frac{\xi+1}{2} \quad-1 \leq \xi \leq 1,
$$

where $L$ is the scaling parameter used to invoke the boundary condition at infinity. We use the popular Gauss-Lobatto collocation points [29,31] to define the Chebyshev nodes in $[-1,1]$, namely:

$$
\xi_{j}=\cos \frac{\pi j}{N} \quad-1 \leq \xi \leq 1, \quad j=0,1,2, \ldots, N,
$$

where $N$ is the number of collocation points. The variable $f_{i, 0}$ is approximated by the interpolating polynomial in terms of its values at each of the collocation points by employing the truncated Chebyshev series of the form:

$$
f_{i, 0}(\xi)=\sum_{k=0}^{N} f_{i, 0}\left(\xi_{k}\right) T_{k}\left(\xi_{j}\right), \quad j=0,1, \ldots, N
$$

where $T_{k}$ is the $k$ th Chebyshev polynomial. Derivatives of the variables at the collocation points may be represented by

$$
\frac{d^{s} f_{i, 0}}{d \eta^{s}}=\sum_{k=0}^{N} \mathbf{D}_{j k}^{s} f_{i, 0}\left(\xi_{k}\right), \quad j=0,1, \ldots, N,
$$

where $s$ is the order of differentiation and $\mathbf{D}=\frac{2}{L} \mathcal{D}$, with $\mathcal{D}$ being the Chebyshev spectral differentiation matrix (see, for example $[29,31]$ ) whose entries are defined as

$$
\begin{aligned}
& \mathcal{D}_{j k}=\frac{c_{j}}{c_{k}} \frac{(-1)^{j+k}}{\xi_{j}-\xi_{k}} \quad j \neq k ; j, k=0,1, \ldots, N, \\
& \mathcal{D}_{k k}=-\frac{\xi_{k}}{2\left(1-\xi_{k}^{2}\right)} \quad k=1,2, \ldots, N-1, \\
& \mathcal{D}_{00}=\frac{2 N^{2}+1}{6}=-\mathcal{D}_{N N} .
\end{aligned}
$$

Substituting Equations $2.20-2.21$ in $2.15-2.16$ gives

$$
\mathbf{A}_{i-1} \mathbf{F}_{i, 0}=\mathbf{R}_{i-1},
$$

subject to

$$
f_{i, 0}\left(\xi_{N}\right)=0, \quad \sum_{k=0}^{N} \mathbf{D}_{N k} f_{i, 0}\left(\xi_{k}\right)=0, \quad \sum_{k=0}^{N} \mathbf{D}_{0 k} f_{i, 0}\left(\xi_{k}\right)=0,
$$


where

$$
\begin{aligned}
& \mathbf{A}_{i-1}=\mathbf{D}^{3}+\mathbf{a}_{1, i-1} \mathbf{D}^{2}+\mathbf{a}_{2, i-1} \mathbf{D}+\mathbf{a}_{3, i-1}, \\
& \mathbf{F}_{i, 0}=\left[f_{i, 0}\left(\xi_{0}\right), f_{i, 0}\left(\xi_{1}\right), \ldots, f_{i, 0}\left(\xi_{N}\right)\right]^{T}, \\
& \mathbf{R}_{i-1}=\left[r_{i-1}\left(\xi_{0}\right), r_{i-1}\left(\xi_{1}\right), \ldots, r_{i-1}\left(\xi_{N}\right)\right]^{T}, .
\end{aligned}
$$

In the above definitions, $T$ stands for transpose and $\mathbf{a}_{k, i-1}(k=1,2,3)$ denotes a diagonal matrix of size $(N+1) \times(N+1)$. The boundary condition $f_{i}\left(\xi_{N}\right)=0$ is implemented by deleting last row and last column of $\mathbf{A}_{i-1}$, and deleting the last rows of $\mathbf{F}_{i, 0}$ and $\mathbf{R}_{i-1}$. The derivative boundary conditions in (2.24) are then imposed on the resulting first row and last row of $\mathbf{A}_{i-1}$ and setting the first and last rows of $\mathbf{F}_{i, 0}$ and $\mathbf{R}_{i-1}$ to be zero. The solutions for $f_{i .0}(\xi)$ are then obtained from soloving

$$
\mathbf{F}_{i, 0}=\mathbf{A}_{i-1}^{-1} \mathbf{R}_{i-1} .
$$

In a similar manner, applying the Chebyshev spectral transformation on the higher order deformation equations (2.12)-(2.13) gives

$$
\mathbf{A F}_{i, m}=\left(\chi_{m}+\hbar\right) \mathbf{A F}_{i, m-1}-\hbar\left(1-\chi_{m}\right) \mathbf{R}_{i-1}+\hbar \mathbf{P}_{i, m-1}
$$

subject to the boundary conditions

$$
f_{i, m}\left(\xi_{N}\right)=0, \quad \sum_{k=0}^{N} \mathbf{D}_{N k} f_{i, m}\left(\xi_{k}\right)=0, \quad \sum_{k=0}^{N} \mathbf{D}_{0 k} f_{i, m}\left(\xi_{k}\right)=0,
$$

where $\mathbf{A}_{i-1}$ and $\mathbf{R}_{i-1}$, are as defined in (2.25) and (2.27), respectively, and

$$
\begin{aligned}
& \mathbf{F}_{i, m}=\left[f_{i, m}\left(\xi_{0}\right), f_{i, m}\left(\xi_{1}\right), \ldots, f_{i, m}\left(\xi_{N}\right)\right]^{T}, \\
& \mathbf{P}_{i, m-1}=c_{1} \sum_{j=0}^{m-1} \mathbf{F}_{i, j}\left(\mathbf{D}^{2} \mathbf{F}_{i, m-1-j}\right)+c_{2} \sum_{j=0}^{m-1}\left(\mathbf{D} F_{i, j}\right)\left(\mathbf{D F}_{i, m-1-j}\right) .
\end{aligned}
$$

To implement the boundary condition $f_{i, m}\left(\xi_{N}\right)=0$, we delete the last rows of $\mathbf{P}_{i, m-1}$ and $\mathbf{R}_{i-1}$ and delete the last row and the last column of $\mathbf{A}_{i-1}$ in (2.29). The other boundary conditions in (2.30) are imposed on the first and the last rows of the modified $\mathbf{A}_{i-1}$ matrix on the left side of the equal sign in (2.29). The first and the last rows of the modified $\mathbf{A}_{i-1}$ matrix on the right side of the equal sign in (2.29) are then set to be zero. This results in the following recursive formula for $m \geq 1$ :

$$
\mathbf{F}_{i, m}=\left(\chi_{m}+\hbar\right) \mathbf{A}_{i-1}^{-1} \tilde{\mathbf{A}}_{i-1} \mathbf{F}_{m-1}+\hbar \mathbf{A}_{i-1}^{-1}\left[\mathbf{P}_{i, m-1}-\left(1-\chi_{m}\right) \mathbf{R}_{i-1}\right],
$$

where $\tilde{\mathbf{A}}_{i-1}$ is the modified matrix $\mathbf{A}_{i-1}$ after incorporating the boundary conditions (2.30). Thus, starting from the initial approximation, which is obtained from (2.28), higher-order approximations $f_{i, m}(\xi)$ for $m \geq 1$, can be obtained through the recursive formula (2.33).

The solutions for $f_{i}$ are then generated using the solutions for $f_{\mathrm{i}, m}$ as follows:

$$
f_{i}=f_{i, 0}+f_{i, 1}+f_{i, 2}+f_{i, 3}+f_{i, 4}+\cdots+f_{i, m} .
$$


Table 1 Order $[i, m]$ ISHAM approximate results for $f^{\prime \prime}(0)$ of the Blasius boundary layer flow (Example 1) using $L=30, \hbar=-1$ and $N=80$

\begin{tabular}{ccccccc}
\hline $\boldsymbol{m}$ & $\mathbf{1}$ & $\mathbf{2}$ & $\mathbf{3}$ & $\mathbf{4}$ & $\mathbf{1 0}$ & $\mathbf{1 5}$ \\
$\boldsymbol{i}$ & & & & & & \\
\hline 1 & 0.33849743 & 0.33398878 & 0.33272105 & 0.33230382 & 0.33205863 & 0.33205736 \\
2 & 0.33205889 & 0.33205734 & 0.33205734 & 0.33205734 & 0.33205734 & 0.33205734 \\
3 & 0.33205734 & 0.33205734 & 0.33205734 & 0.33205734 & 0.33205734 & 0.33205734 \\
4 & 0.33205734 & 0.33205734 & 0.33205734 & 0.33205734 & 0.33205734 & 0.33205734 \\
5 & 0.33205734 & 0.33205734 & 0.33205734 & 0.33205734 & 0.33205734 & 0.33205734 \\
\hline
\end{tabular}

The $[i, m]$ approximate solution for $f(\eta)$ is then obtained by substituting $f_{i}$ (obtained from 2.34) in equation 2.3.

\section{Results and discussion}

Table 1 shows the values of $f^{\prime}(0)$ at different orders $[i, m]$ of the ISHAM approximation for the Blasius boundary layer flow when $L=30, \hbar=-1$ and $N=80$. It is worth noting here that the numerical solution given by Howarth [32] is $f^{\prime \prime}(0)=0.332057$, while the numerical result by the Matlab bvp4c routine is $f^{\prime \prime}(0)=0.33205734$. Asaithambi [33] found this number correct to nine decimal positions as 0.332057336 . It is evident that the ISHAM converges to the numerical result at orders [3,1] and $[2,2]$. Moreover, Table 1 shows that the ISHAM solution converges to the accurate solution of Howarth and the bvp $4 \mathrm{C}$ result faster than the original SHAM results of which are those given in the first row of Table 1 (for the case when $i=1$ ).

In general, at order $[i, m], i$ is the number of improvements of the initial approximation $f_{0}(\eta)$ for $f(\eta)$, and $m$ is the number of improvements of the initial guess $f_{q, 0}(\eta) ; q$ $=1,2, \ldots, i$, for each application of the ISHAM. Table 2 gives a sense of the convergence rate of the ISHAM when compared with the numerical method for the Blasius problem at different values of $\eta$. In all the instances, convergence of the ISHAM is achieved at the second order.

Table 3 gives the values of $f^{\prime \prime}(0)$ obtained used the ISHAM and the numerical method for various values of $\beta$ for the Falkner-Skan boundary layer problem. Full convergence is again achieved at order $[2,2]$ for all the parameter values.

Table 2 Comparison between the $[m, m]$ ISHAM results and the bvp $4 \mathrm{C}$ numerical results for the velocity pro le $f^{\prime}(\eta)$ at selected values of $\eta$ for the Blasius boundary layer flow (Example 1) using $L=30, \hbar=-1$ and $N=200$

\begin{tabular}{cccccc}
\hline $\boldsymbol{\eta}$ & {$[\mathbf{1 , 1 ]}$} & {$[\mathbf{2 , 2}]$} & {$[\mathbf{3 , 3}]$} & {$[\mathbf{4 , 4 ]}$} & Numerical \\
\hline 0.0 & 0.0000000 & 0.0000000 & 0.0000000 & 0.0000000 & 0.0000000 \\
0.4 & 0.1353503 & 0.1327642 & 0.1327642 & 0.1327642 & 0.1327642 \\
0.8 & 0.2699826 & 0.2647092 & 0.2647092 & 0.2647092 & 0.2647091 \\
1.6 & 0.5279353 & 0.5167568 & 0.5167568 & 0.5167568 & 0.5167568 \\
2.0 & 0.6436159 & 0.6297657 & 0.6297657 & 0.6297657 & 0.6297657 \\
3.0 & 0.8609681 & 0.8460445 & 0.8460445 & 0.8460445 & 0.8460444 \\
4.0 & 0.9635769 & 0.9555182 & 0.9555182 & 0.9555182 & 0.9555182 \\
5.0 & 0.9937558 & 0.9915420 & 0.9915420 & 0.9915420 & 0.9915419 \\
6.0 & 0.9992643 & 0.9989729 & 0.9989729 & 0.9989729 & 0.9989729 \\
8.0 & 0.9999880 & 0.9999963 & 0.9999963 & 0.9999963 & 0.9999963 \\
10.0 & 0.9999991 & 1.0000000 & 1.0000000 & 1.0000000 & 1.0000000 \\
\hline
\end{tabular}


Table 3 Order $[m, m]$ ISHAM approximate results for $f^{\prime \prime}(0)$ of the Falkner-Skan boundary layer flow (Example 2) using $L=30, \hbar=-1$ and $N=80$

\begin{tabular}{cccccc}
\hline $\boldsymbol{\beta}$ & {$[\mathbf{1 , 1}]$} & {$[\mathbf{2 , 2}]$} & {$[\mathbf{3 , 3}$} & {$[\mathbf{4 , 4 ]}$} & Numerical \\
\hline 0.4 & 0.85435667 & 0.85442123 & 0.85442123 & 0.85442123 & 0.85442123 \\
0.8 & 1.11956168 & 1.12026766 & 1.12026766 & 1.12026766 & 1.12026766 \\
1.2 & 1.33311019 & 1.33572147 & 1.33572147 & 1.33572147 & 1.33572147 \\
1.6 & 1.51553054 & 1.52151400 & 1.52151400 & 1.52151400 & 1.52151400 \\
2.0 & 1.67637221 & 1.68721817 & 1.68721817 & 1.68721817 & 1.68721817 \\
\hline
\end{tabular}

Table 4 Order $[m, m]$ ISHAM approximate results for the velocity profile $f^{\prime}(\eta)$ of the MHD boundary layer flow (Example 3 ) when $M=10$ using $L=10, \hbar=-1$ and $N=200$

\begin{tabular}{cccccccc}
\hline $\boldsymbol{\eta}$ & \multicolumn{3}{c}{$\boldsymbol{f}^{\prime}(\boldsymbol{\eta})$} & \multicolumn{3}{c}{ Exact } & \multicolumn{3}{c}{ Absolute error } \\
\cline { 2 - 3 } \cline { 7 - 8 } & {$[\mathbf{1 , 1 ]}$} & {$[\mathbf{2 , 2}]$} & {$[\mathbf{3 , 3}]$} & & {$[\mathbf{1 , 1}]$} & {$[\mathbf{2 , 2}]$} & {$[\mathbf{3 , 3}]$} \\
\hline 0.0 & 1.00000000 & 1.00000000 & 1.00000000 & 1.000000000 & 0.00000000 & 0.000000000 & 0.00000000 \\
0.5 & 0.19106051 & 0.19046007 & 0.19046007 & 0.19046013 & 0.00060038 & 0.000000006 & 0.00000006 \\
1.0 & 0.03731355 & 0.03627506 & 0.03627506 & 0.03627506 & 0.00103849 & 0.000000000 & 0.00000000 \\
1.5 & 0.00795438 & 0.00690893 & 0.00690893 & 0.00690895 & 0.00104543 & 0.000000002 & 0.00000002 \\
2.0 & 0.00212716 & 0.00131588 & 0.00131588 & 0.00131588 & 0.00081128 & 0.000000000 & 0.00000000 \\
2.5 & 0.00080280 & 0.00025062 & 0.00025062 & 0.00025062 & 0.00055218 & 0.000000000 & 0.00000000 \\
3.0 & 0.00040021 & 0.000047773 & 0.00004773 & 0.00004773 & 0.00035248 & 0.000000000 & 0.00000000 \\
3.5 & 0.00022752 & 0.000000909 & 0.00000909 & 0.00000909 & 0.00021843 & 0.000000000 & 0.00000000 \\
4.0 & 0.00013536 & 0.000000173 & 0.00000173 & 0.00000173 & 0.00013363 & 0.000000000 & 0.00000000 \\
5.0 & 0.00004944 & 0.000000006 & 0.00000006 & 0.00000006 & 0.00004938 & 0.000000000 & 0.00000000 \\
6.0 & 0.00001818 & 0.000000000 & 0.00000000 & 0.00000000 & 0.00001818 & 0.000000000 & 0.00000000 \\
\hline
\end{tabular}

Table 5 Order $[m, m]$ ISHAM approximate results for $f^{\prime \prime}(\eta)$ of the MHD boundary layer flow (Example 3) for different values of $M$ using $L=10, \hbar=-1$ and $N=200$

\begin{tabular}{|c|c|c|c|c|c|}
\hline \multirow[t]{2}{*}{$M$} & \multicolumn{2}{|c|}{$f^{\prime \prime}(0)$} & \multirow[t]{2}{*}{ Exact } & \multicolumn{2}{|c|}{ Absolute error } \\
\hline & {$[1,1]$} & {$[2,2]$} & & {$[1,1]$} & {$[2,2]$} \\
\hline 5 & -2.44812872 & -2.44948974 & -2.44948974 & 0.00136102 & 0.00000000 \\
\hline 10 & -3.31554301 & -3.31662479 & -3.31662479 & 0.00108178 & 0.00000000 \\
\hline 20 & -4.58188947 & -4.58257570 & -4.58257569 & 0.00068622 & 0.00000001 \\
\hline 50 & -7.14113929 & -7.14142843 & -7.14142843 & 0.00028914 & 0.00000000 \\
\hline 100 & -10.04974330 & -10.04987562 & -10.04987562 & 0.00013232 & 0.00000000 \\
\hline 200 & -14.17739008 & -14.17744688 & -14.17744688 & 0.00005680 & 0.00000000 \\
\hline 500 & -22.38301286 & -22.38302928 & -22.38302929 & 0.00001643 & 0.00000001 \\
\hline 1000 & -31.63857773 & -31.63858404 & -31.63858404 & 0.00000631 & 0.00000000 \\
\hline
\end{tabular}

For the MHD boundary layer problem, Tables 4 and 5 illustrate the exact and approximate values of $f(\eta)$ and $f^{\prime \prime}(0)$ at different values of $\eta$ and the magnetic parameter $M$, respectively. The absolute errors in the approximations are also given. The tables show that the ISHAM converges rapidly with marginal or no errors after order $[2,2]$.

\section{Conclusion}

In this article, we have proposed an ISHAM for solving general nonlinear differential equations. This novel technique was compared against both numerical approximations and the MATLAB bvp4c routine for solving Falkner-Skan and MHD boundary layer problems. The results demonstrate the relatively more rapid convergence of the ISHAM, and they show that the ISHAM is highly accurate. 


\section{Abbreviations}

HAM: homotopy analysis method; ISHAM: improved spectral-homotopy analysis method; MHD: magnetohydrodynamic; SHAM: spectral-homotopy analysis.

\section{Acknowledgements}

The authors wish to acknowledge financial support from the University of Swaziland, University of KwaZulu-Natal, University of Venda, and the National Research Foundation (NRF).

\section{Author details}

'Department of Mathematics, University of Swaziland, Private Bag 4, Kwaluseni, Swaziland ${ }^{2}$ School of Mathematical Sciences, University of KwaZulu-Natal, Private Bag X01, Scottsville 3209, Pietermaritzburg, South Africa ${ }^{3}$ Department of Mathematics, University of Venda, Private Bag X5050, Thohoyandou 0950, South Africa

\section{Authors' contributions}

SSM developed the Matlab codes and generated the results. GTM and PS conceived of the study and formulated the problem. SS participated in the analysis of the results and manuscript coordination. All authors typed, read and approved the final manuscript.

\section{Competing interests}

The authors declare that they have no competing interests.

Received: 10 November 2010 Accepted: 22 June 2011 Published: 22 June 2011

\section{References}

1. Blasius, H: Grenzschichten in Flussigkeiten mit kleiner Reibung. Z Math Phys. 56, 1-37 (1908)

2. Ahmad, F, Al-Barakati, WH: An analytic solution of the Blasius problem. Commun Nonlinear Sci Numer Simul. 14 1020-1024 (2009)

3. Alizadeh-Pahlavan, A, Borjian-Boroujeni, S: On the analytic solution of viscous fluid flow past a flat plate. Phys Lett A. 372, 3678-3682 (2008). doi:10.1016/j.physleta.2008.02.050

4. Parand, K, Shahini, M, Dehghan, M: Solution of a laminar boundary layer flow via a numerical method. Commun Nonlinear Sci Numer Simulat. 15, 360-367 (2010). doi:10.1016/j.cnsns.2009.04.007

5. Yun, Bl: Intuitive approach to the approximate analytical solution for the Blasius problem. Appl Math Comput. 208, 156-164 (2009). doi:10.1016/j.amc.2008.11.028

6. Falkner, VM, Skan, SW: Some approximate solutions of the boundary layer equations. Philos Mag. 12, $865-896$ (1931)

7. Harris, SD, Ingham, DB, Pop, I: Unsteady heat transfer in impulsive Falkner-Skan flows: constant wall temperature case. Eur J Mech B. 21, 447-468 (2002). doi:10.1016/S0997-7546(02)01193-7

8. Padé, O: On the solution of Falkner-Skan equations. J Math Anal Appl. 285, 264-274 (2003). doi:10.1016/S0022-247X(03) 00402-5

9. Pantokratoras, A: The Falkner-Skan flow with constant wall temperature and variable viscosity. Int J Thermal Sci. 45 , 378-389 (2006). doi:10.1016/j.ijthermalsci.2005.06.004

10. Rashidi, MM: The modified differential transform method for solving MHD boundary-layer equations. Comput Phys Commun. 180, 2210-2217 (2009). doi:10.1016/j.cpc.2009.06.029

11. Parand, K, Rezai, AR, Ghaderi, SM: An approximate solution of the MHD Falkner-Skan flow by Hermite functions pseudospectral method. Commun Nonlinear Sci Numer Simulat. (2010)

12. Asaithambi, A: A second-order finite-difference method for the Falkner-Skan equation. Appl Math Comput. 156, 779-786 (2004). doi:10.1016/j.amc.2003.06.020

13. Cortell, R: Numerical solutions of the classical Blasius flat-plate problem. Appl Math Comput. 170, 706-710 (2005). doi:10.1016/j.amc.2004.12.037

14. Asaithambi, A: Numerical solution of the Falkner-Skan equation using piecewise linear functions. Appl Math Comput. 159, 267-273 (2004). doi:10.1016/j.amc.2003.10.047

15. Elgazery, NS: Numerical solution for the Falkner-Skan equation. Chaos Soliton Fract. 35, 738-746 (2008). doi:10.1016/j. chaos.2006.05.040

16. Wang, L: A new algorithm for solving classical Blasius equation. Appl Math Comput. 157, 1-9 (2004). doi:10.1016/j. amc.2003.06.011

17. Abbasbandy, S: A numerical solution of Blasius equation by Adomian's decomposition method and comparison with homotopy perturbation method. Chaos Soliton Fract. 31, 257-260 (2007). doi:10.1016/j.chaos.2005.10.071

18. Kuo, B: Heat analysis for the Falkner-Skan wedge flow by the differential transformation method. Int J Heat Mass Transfer. 48, 5036-5046 (2005). doi:10.1016/j.ijheatmasstransfer.2003.10.046

19. Wazwaz, A: The variational iteration method for solving two forms of Blasius equation on a half-infinite domain. Appl Math Comput. 188, 485-491 (2007). doi:10.1016/j.amc.2006.10.009

20. Liao, SJ: Beyond Perturbation: Introduction to Homotopy Analysis Method. Chapman \& Hall/CRC Press (2003)

21. Yao, B, Chen, J: A new analytical solution branch for the Blasius equation with a shrinking sheet. Appl Math Comput. 215, 1146-1153 (2009). doi:10.1016/j.amc.2009.06.057

22. Yao, B, Chen, J: Series solution to the Falkner-Skan equation with stretching boundary. Appl Math Comput. 215 1146-1153 (2009). doi:10.1016/j.amc.2009.06.057

23. Yao, B: Approximate analytical solution to the Falkner-Skan wedge flow with the permeable wall of uniform suction. Commun Nonlinear Sci Numer Simulat. 14, 3320-3326 (2009). doi:10.1016/j.cnsns.2009.01.014

24. Motsa, SS, Sibanda, P, Shateyi, S: A new spectral-homotopy analysis method for solving a nonlinear second order BVP. Commun Nonlinear Sci Numer Simulat. 15, 2293-2302 (2010). doi:10.1016/j.cnsns.2009.09.019

25. Motsa, SS, Sibanda, P, Awad, FG, Shateyi, S: A new spectral-homotopy analysis method for the MHD Jeffery-Hame problem. Comput Fluids. 39, 1219-1225 (2010). doi:10.1016/j.compfluid.2010.03.004 
26. Makukula, Z, Motsa, SS, Sibanda, P: On a new solution for the viscoelastic squeezing flow between two parallel plates. J Adv Res Appl Math. 2(4):31-38 (2010). doi:10.5373/jaram.455.060310

27. Makukula, ZG, Sibanda, P, Motsa, SS: A novel numerical technique for two-dimensional laminar flow between two moving porous walls. Math Problems Eng. Article ID 528956, 15 (2010)

28. Shateyi, S, Motsa, SS: Variable viscosity on magnetohydrodynamic fluid flow and heat transfer over an unsteady stretching surface with Hall effect. Boundary Value Problems. 2010, 1-20 (2010)

29. Canuto, C, Hussaini, MY, Quarteroni, A, Zang, TA: Spectral Methods in Fluid Dynamics. Springer-Verlag, Berlin (1988)

30. Don, WS, Solomonoff, A: Accuracy and speed in computing the Chebyshev Collocation Derivative. SIAM I Sci Comput. 16(6):1253-1268 (1995). doi:10.1137/0916073

31. Trefethen, LN: Spectral Methods in MATLAB. SIAM. (2000)

32. Howarth, L: On the solution of the laminar boundary layer equations. Proc R Soc Lond A. 164, 547-579 (1938). doi:10.1098/rspa.1938.0037

33. Asaithambi, A: Solution of the Falkne-Skan equation by recursive evaluation of Taylor coefficients. J Comput Appl Math. 176, 203-14 (2005). doi:10.1016/j.cam.2004.07.013

doi:10.1186/1687-2770-2011-3

Cite this article as: Motsa et al: An improved spectral homotopy analysis method for solving boundary layer problems. Boundary Value Problems 2011 2011:3.

\section{Submit your manuscript to a SpringerOpen ${ }^{\circ}$} journal and benefit from:

Convenient online submission

- Rigorous peer review

- Immediate publication on acceptance

- Open access: articles freely available online

- High visibility within the field

- Retaining the copyright to your article

Submit your next manuscript at $\gg$ springeropen.com 